\title{
SDU
}

\section{Observable implications of the conditional CAPM}

\author{
by
}

Thiago de Oliveira Souza

Discussion Papers on Business and Economics

No. $13 / 2020$

FURTHER INFORMATION

Department of Business and Economics

Faculty of Business and Social Sciences University of Southern Denmark Campusvej 55, DK-5230 Odense M

Denmark 


\title{
Observable implications of the conditional CAPM
}

\author{
Thiago de Oliveira Souza \\ University of Southern Denmark; \\ Danish Finance Institute
}

\begin{abstract}
The derivation of observable implications of the conditional CAPM theory often includes the joint (internally inconsistent) hypothesis that the stock portfolio used in the tests is the theoretical, mean-variance efficient, market portfolio. The present paper generalizes this derivation by avoiding this joint hypothesis. The generalization reveals that the conditional CAPM plausibly explains asset pricing anomalies, such as the unconditional alphas and betas of momentum, value, and size portfolios, while the unconditional CAPM theory is still rejected by portfolios with negative unconditional betas and positive unconditional alphas. Hence, relaxing this joint assumption does not render the CAPM theory untestable.
\end{abstract}

JEL Code: G11, G12, G14.

Keywords: Conditional CAPM, anomalies, test, proxy, mean-variance frontier.

Department of Business and Economics, University of Southern Denmark, Campusvej 55, 5230 Odense M, Denmark. Email: tsouza@sam.sdu.dk. I would like to thank Campbell Harvey. 


\section{Introduction}

"A statistical test is a probabilistic twist of the mathematical proof by contradiction: If the observed event is sufficiently improbable under the hypothesis, the hypothesis can reasonably be rejected" (Schweder, 2001).

Under the theoretical assumption that the CAPM holds (conditionally or unconditionally), the market portfolio is on the (conditional or unconditional) mean-variance frontier (MVF). Under the additional assumption that the Center for Research in Security Prices (CRSP) value-weighted index is the market portfolio, then the CRSP portfolio must be on the MVF. If the researcher is interested on this joint hypothesis test, it is trivial to simply compare the Sharpe ratio of the market proxy and the Sharpe ratio of the mean variance frontier. Estimated values of the monthly Sharpe ratio of the MVF are at least 0.38 (Ghosh et al., 2016). Even without a formal test, this value can be argued to be implausibly larger than the CRSP portfolio Sharpe ratio, close to 0.11 (Lewellen and Nagel, 2006), and the hypothesis is reasonably rejected. This renders all remaining tests of this joint hypothesis unnecessary.

The present paper is not about this joint hypothesis. The paper is about the (single) hypothesis that the conditional CAPM theory explains asset prices. Different procedures in the literature supposedly test this hypothesis (leading to conflicting conclusions). Among them, the (magnitude) "tests" in Lewellen and Nagel (2006) have been particularly influential in rejecting the theory. I contribute to this discussion by (i) clarifying that the tests in Lewellen and Nagel (2006) are restatements of the joint test described in the paragraph above and (ii) proposing a modified (magnitude) "test", only for the theory in the CAPM. Hence, the paper is primarily about the testing procedure, not its outcome. However, I also show that the conditional CAPM explains the asset pricing anomalies that Lewellen and Nagel (2006) claim to be unexplained based on similar quantitative assumptions: The differences in the tests are relevant.

Jagannathan and Wang (1996), Lettau and Ludvigson (2001), and many others conclude that conditional CAPM theories in traditional and consumption-based forms explain asset 
prices. ${ }^{1}$ In summary, they judge that the observed correlations between time-varying betas and expected returns from cross sectional regressions are sufficiently improbable under the assumption that the conditional CAPM theory does not hold. So they conclude that it holds.

Lewellen and Nagel (2006) disagree with the test procedure (and also with the conclusion): They argue that these tests only consider the qualitative implications of the conditional CAPM, instead of performing a full, quantitative test of the theory. Hence, they propose two different tests.

In their main tests, Lewellen and Nagel (2006) conclude that the CAPM intercepts observed in unconditional time-series regressions are sufficiently improbable under the hypothesis that the conditional CAPM theory holds, and reject the theory. The conclusion is mostly justified by the argument that "ignoring all variation in beta has little impact on asset-pricing tests" (Lewellen and Nagel, 2006, p. 297). Hence, these tests are not based on formal probability calculations and compared to a given distribution: The parameters are simply claimed to be "sufficiently implausible", given all reasonable quantitative estimates of certain parameters. I highlight this aspect of their test, because I use the same procedure in my own test, which means that neither is a formal test in a strict sense.

The second group of tests is based on a rolling window procedure similar to Ferson and Harvey (1999): Lewellen and Nagel (2006) conclude that the average CAPM intercepts obtained in several linear regressions in small sample periods are sufficiently improbable under the hypothesis that the conditional CAPM theory holds, and reject the theory once again.

Since then, these two have largely become the standard tests of the conditional CAPM theory. For example, even conclusions that contradict the one in Lewellen and Nagel (2006),

\footnotetext{
${ }^{1}$ A short list of similar results includes Wang (2003), Lustig and Nieuwerburgh (2005), Petkova and Zhang (2005), Acharya and Pedersen (2005), Ang et al. (2006), Santos and Veronesi (2006), Piazzesi et al. (2007), Bali (2008), Choi (2013), Lettau et al. (2014), Bali et al. (2017), Farago and Tédongap (2018), Hasler and Martineau (2019), and Atanasov et al. (2020). On the other hand, the GMM tests in Harvey (1989) suggest that the conditional CAPM does not hold, for example.
} 
as Boguth et al. (2011), still tend to be based on the relation between unconditional and conditional CAPM parameters derived by Lewellen and Nagel (2006).

The central point of the present paper is that the derivation of the relation between unconditional and conditional CAPM parameters in Lewellen and Nagel (2006) relies on two contradicting assumptions: The theoretical assumption is that the CAPM holds conditionally; the empirical assumption is that the CRSP portfolio is the exact theoretical market portfolio in the CAPM. This empirical assumption already contradicts the hypothesis that the CAPM theory explains asset prices, which is exactly what Lewellen and Nagel (2006) claim to test: At minimum, the market portfolio theoretically includes human capital, private businesses, farms, real estate, bonds, and international assets. None of these is included in the CRSP index. ${ }^{2}$ This assumption is also empirically false because the CRSP portfolio is arguably not on the MVF. So even if the CAPM theory holds, the joint hypothesis is false. In order to avoid this inconsistency, I create a (magnitude) test for the conditional CAPM similat to the one created by Lewellen and Nagel (2006), but allowing for the stock portfolio used in the empirical analysis of the CAPM to be just a proxy for the true market portfolio.

Incorporating this theoretical feature of the CAPM into the test can be challenging: If the true market portfolio is completely unobservable, the effect on the estimated parameters is unknown (Kandel and Stambaugh, 1995), every version of the CAPM is untestable (Roll, 1977), and therefore the theory cannot be regarded as scientific (Campbell, 2017). I avoid this outcome by restricting the market proxy returns to contain a simple idiosyncratic component, uncorrelated with all observable quantities. In summary, the returns on the proxy portfolio have a classic measurement error relative to the market portfolio. This assumption is enough to make the test internally consistent with the CAPM, while keeping the theory scientifically valid.

Next, I revisit Lewellen and Nagel (2006) and show that the conditional CAPM in fact explains asset pricing anomalies, such as value (Fama and French, 1992) and momentum

\footnotetext{
${ }^{2}$ For the consumption-based version of the CAPM, in which GDP growth is used, the assumption is even stronger because consumption is notoriously difficult to measure and usually (poorly) approximated by expenditures.
} 
(Jegadeesh and Titman, 1993), based on mostly the same quantitative assumptions that they make to reject the theory. The exercise also confirms that the conditional version of the CAPM can explain asset pricing anomalies that the unconditional version cannot. This conclusion also reveals that the recommendation in Lewellen and Nagel (2006) to ignore all variation in beta is not innocuous: Unconditional CAPM regressions cannot be used to draw conclusions about the conditional CAPM. At minimum, it is not clear that the testing procedure in Lewellen and Nagel (2006) is superior to the several cross-sectional tests in Lettau and Ludvigson (2001), Jagannathan and Wang (1996), or Harvey (1989), for example.

\subsection{Inference about the conditional and unconditional CAPM}

Any least squares regression based on a variable measured with error provides inconsistent estimates of the true coefficients of interest. A CAPM estimation based on a proxy for the true market portfolio is an example of such a regression: The estimated proxy beta has an attenuation bias with respect to the true beta. Given the Sharpe ratio of the MVF, of at least 0.38 (Ghosh et al., 2016), and the market proxy Sharpe ratio, close to 0.11 (Lewellen and Nagel, 2006), the bias is severe: If we assume that the CAPM holds unconditionally, for the sake of illustration, these numbers imply that the estimated proxy beta is around $7 \%$ of the true beta and the proxy alpha is $93 \%$ of the average premium on the asset. Therefore, even the unconditional CAPM generates the flat relation between (proxy) betas and expected returns observed empirically (Fama and French, 1992). ${ }^{3}$ However, this result still renders the unconditional CAPM inconsistent with the existence of portfolios that have negative proxy betas and positive average returns in general. All the 11 "anomalies" in Stambaugh et al. (2012) and even value after 1960 are examples of such portfolios. ${ }^{4}$

\footnotetext{
${ }^{3}$ The flat relation between betas and expected returns is also discussed in Stambaugh (1982), Roll and Ross (1994), and Andrei et al. (2018), for example.

${ }^{4}$ The anomalies in Stambaugh et al. (2012) are momentum (Jegadeesh and Titman, 1993), failure probability (Campbell et al., 2008), financial distress (Ohlson, 1980), net stock issues (Loughran and Ritter, 1995), composite equity issues (Daniel and Titman, 2006), net operating assets (Hirshleifer et al., 2004), accruals (Sloan, 1996), gross-profitability (Novy-Marx, 2013), asset growth (Cooper et al., 2008), return on assets (Fama and French, 2006), and investment-to-assets (Titman et al., 2004).
} 
Hence, the existence of these portfolios can be used as evidence to reject the theory in the unconditional CAPM even after we consider that the stock portfolio is not the true market portfolio.

Under the theoretical assumption that the CAPM holds conditionally, I show that the unconditional proxy beta is approximately determined by (i) the attenuated true expected beta, added to a term that depends positively on both (ii) the covariance between conditional betas and the market portfolio returns and (iii) the covariance between conditional betas and the variance of returns on the market portfolio. The unconditional proxy alpha is approximately $93 \%$ of the expected conditional premium on the asset plus a term that depends positively on the covariance in (ii) and negatively on (iii). This explains why some portfolios have negative proxy betas and positive proxy alphas. Below is a group of values for which the conditional CAPM explains the premiums that Lewellen and Nagel (2006) claim to be impossible to explain.

When the two covariances in the previous paragraph are plausibly negative for momentum portfolios, they reinforce each other and generate an unconditional proxy beta of -0.5 . For these same values, the net effect that the covariances have on the unconditional proxy alpha is also negative. Yet, the unconditional proxy alpha is positive, $1.01 \%$ per month, because the average conditional momentum premium is relatively large, even if the portfolio has a relatively low risk: If constant, the true and unobservable expected beta would correspond to a market proxy beta between 0.29 and 0.39 . For value portfolios, these covariances have opposite signs: The positive covariance between conditional betas and the market portfolio returns contributes to increase the unconditional proxy alpha to $0.59 \%$. The negative covariance between conditional betas and the variance of returns on the market portfolio contributes to lower the unconditional proxy beta to -0.25 , while the equivalent to the expected portfolio beta (on the market proxy) is in fact positive, between 0.01 and 0.08. Given these quantities, the conditional CAPM explains both value and momentum. 


\section{The conditional CAPM}

Let $R_{i, t}$ be the excess return on asset $i, R_{M, t}$ be the excess return on the (true) market portfolio, and $R_{P, t}$ be the excess return on the (market) proxy portfolio. The joint distribution of $R_{i, t}$ and $R_{M, t}$ and the joint distribution of $R_{i, t}$ and $R_{P, t}$ change over time with well-defined conditional and unconditional moments and the conditional CAPM holds. The $t$ subscripts indicate conditional moments for period $t$ given information at $t-1$ : The conditional risk premium and standard deviation of the market portfolio are $g_{t}$ and $s_{t}$, and the (true) conditional beta of the stock is $b_{t}$. In terms of notation, Greek letters usually refer to the market proxy: The conditional risk premium and standard deviation of the proxy portfolio are $\gamma_{t}$ and $\sigma_{t}$, and the conditional (proxy) beta of the stock with respect to the returns on this proxy is $\beta_{t}$. The corresponding unconditional moments are denoted $g, s_{M}, b^{u}, \gamma$, $\sigma_{P}$, and $\beta^{u}$. The unconditional betas, $\beta^{u}$ and $b^{u}$, are usually different from the expected conditional betas, denoted $\beta \equiv \mathrm{E}\left[\beta_{t}\right]$ and $b \equiv \mathrm{E}\left[b_{t}\right]$.

The return on the proxy portfolio contains the idiosyncratic component $v_{t}$,

$$
R_{P, t}=R_{M, t}+v_{t},
$$

which I assume to have mean zero and variance $\sigma_{v, t}^{2}$. I also restrict this idiosyncratic component to be conditionally independent of the betas of individual assets (in addition to being independent of the return on the market),

$$
v_{t} \Perp b_{t}, v_{t} \Perp R_{M, t} \quad \forall t .
$$

Naturally, the market proxy is inside the MVF, but the simplified formulation in Eq. (1) implies that it has the same conditional and unconditional mean returns as the market portfolio, $g=\gamma$ and $g_{t}=\gamma_{t}$. According to the conditional CAPM, the expected return on the asset is proportional to its conditional beta on the market portfolio,

$$
\mathrm{E}_{t-1}\left[R_{i, t}\right]=b_{t} g_{t},
$$


implying that the realized returns are given by (Lewellen and Nagel, 2006),

$$
R_{i, t}=b_{i, t} R_{M, t}+e_{t}
$$

This is the regression that we would like to estimate, which is unfeasible because the return on the market is unobservable.

A second-best alternative would be to estimate an instantaneous regression on the (observable) market proxy returns,

$$
R_{i, t}=\alpha_{t}+\beta_{t} R_{P, t}+e_{p, t}
$$

But, naturally, this estimation is also unfeasible because of the time-varying coefficients. Hence, as a third-best, we obtain $\beta^{u}$ and $\alpha^{u}$ from unconditional least squares regressions of the asset returns on the market proxy returns during a given sample period, ${ }^{5}$

$$
R_{i, t}=\alpha^{u}+\beta^{u} R_{P, t}+\epsilon_{t},
$$

with proxy beta given by

$$
\beta^{u}=\frac{\operatorname{cov}\left(R_{i, t}, R_{P, t}\right)}{\operatorname{var}\left(R_{P, t}\right)}=\frac{\operatorname{cov}\left(R_{i, t}, R_{M t}\right)}{\operatorname{var}\left(R_{P, t}\right)} \neq \frac{\operatorname{cov}\left(R_{i, t}, R_{M t}\right)}{\operatorname{var}\left(R_{M t}\right)}=b^{u},
$$

where the second equality follows from the assumption that the measurement error in Eq. (1) is uncorrelated with the quantities in Eq. (4), in Eq. (2), and $b^{u}$ is the slope of a hypothetical regression that replaces the market proxy returns in Eq. (6) by the market returns. In particular, Lewellen and Nagel (2006) assume that unconditional regressions on the market proxy produce coefficients equal to $b^{u}$. However, in addition to the issues related to the unconditional estimation, $\beta^{u}$ has an attenuation bias with respect to $b^{u}$

\footnotetext{
${ }^{5}$ An alternative third best solution is to include another assumption in the CAPM test: The assumption that we know the exact state variables that represent the full information set of the individuals (Lewellen and Nagel, 2006). In this case, we could model conditional betas as linear functions of these state variables (Harvey, 1989; Lettau and Ludvigson, 2001, for example) to obtain an unconditional version of the test, as explained in Cochrane (2005).
} 
proportional to the difference between the variances of the market portfolio and the market proxy.

Eq. (A.4) in the appendix (Lewellen and Nagel, 2006) gives the covariance in Eq. (7). This covariance divided by the variance of the market proxy returns yields

$$
\beta^{u}=b \frac{s_{M}^{2}}{\sigma_{P}^{2}}+\frac{g}{\sigma_{P}^{2}} \operatorname{cov}\left(b_{t}, g_{t}\right)+\frac{1}{\sigma_{P}^{2}} \operatorname{cov}\left(b_{t}, s_{t}^{2}\right)+\frac{1}{\sigma_{P}^{2}} \operatorname{cov}\left[b_{t},\left(g_{t}-g\right)^{2}\right] .
$$

The asset's unconditionally estimated alpha in Eq. (6) is

$$
\alpha^{u} \equiv \mathrm{E}\left[R_{i, t}\right]-\beta^{u} \mathrm{E}\left[R_{P, t}\right]=\mathrm{E}\left[R_{i, t}\right]-\beta^{u} \gamma
$$

and the unconditional expectation of Eq. (4), given by Jagannathan and Wang (1996), is

$$
\mathrm{E}\left[R_{i, t}\right]=b g+\operatorname{cov}\left(b_{t}, g_{t}\right)
$$

Hence, we substitute $\mathrm{E}\left[R_{i, t}\right]$ (and $g=\gamma$ ) to obtain

$$
\alpha^{u}=\gamma\left(b-\beta^{u}\right)+\operatorname{cov}\left(b_{t}, g_{t}\right),
$$

and Eq. (8) to obtain

$$
\alpha^{u}=\gamma b\left(1-\frac{\sigma_{M}^{2}}{\sigma_{P}^{2}}\right)+\left(1-\frac{\gamma^{2}}{\sigma_{P}^{2}}\right) \operatorname{cov}\left(b_{t}, g_{t}\right)-\frac{\gamma}{\sigma_{P}^{2}} \operatorname{cov}\left(b_{t}, s_{t}^{2}\right)-\frac{\gamma}{\sigma_{P}^{2}} \operatorname{cov}\left[b_{t},\left(g_{t}-g\right)^{2}\right] .
$$

Eq. (8) and Eq. (12) are versions of the unconditional CAPM slope and pricing error formulas in Lewellen and Nagel (2006), but modified to make them internally consistent with the CAPM theory being tested. In particular, the formulas in Lewellen and Nagel (2006) ignore the attenuation term in Eq. (8) and the corresponding inflation term in Eq. (12). Their formulas also ignore that the relevant covariance terms involve betas and returns on the true market portfolio, not on the proxy portfolio. 


\subsection{Parameter restrictions in typical regressions}

Probably the most important contribution of Lewellen and Nagel (2006) is to highlight that certain estimates from unconditional CAPM regressions would require "sufficiently improbable" parameters in Eq. (8) and Eq. (12), implying that the conditional CAPM theory could be rejected even without a formal statistical test. This section revisits the analytical exercise in Lewellen and Nagel (2006).

For typical monthly frequency regressions, Lewellen and Nagel (2006) argue that two realistic values are $g=\gamma=0.47 \%$ as the mean of the excess return on the market proxy and $\sigma_{P}=4.5 \%$ as its standard deviation. This implies that the squared Sharpe ratio of the market proxy portfolio is $\frac{\gamma^{2}}{\sigma_{P}^{2}} \approx 0.011$, while $\frac{\gamma}{\sigma_{P}^{2}} \approx 2.32$ and $\frac{1}{\sigma_{P}^{2}} \approx 493.83$. They also argue that the quadratic $\left(\gamma_{t}-\gamma\right)^{2}$ in the last terms in the equations is negligible.

Without the joint assumption that the CRSP portfolio is the exact market portfolio, we also need an estimate for the Sharpe ratio of the true market portfolio. A conservative (out-of-sample) estimate for the monthly MVF Sharpe ratio is 0.38 (Ghosh et al., 2016, Table 12). With this conservative lower bound for the MVF Sharpe ratio, we obtain an upper bound for $\frac{\sigma_{M}^{2}}{\sigma_{p}^{2}} \leq 0.076$. This upper bound generates the lowest attenuation bias. Under this assumption, we approximate $\alpha^{u}$ and $\beta^{u}$ for data in monthly frequency by

$$
\begin{aligned}
& \alpha^{u} \approx 0.43 \% b+0.99 \operatorname{cov}\left(b_{t}, g_{t}\right)-2.32 \operatorname{cov}\left(b_{t}, s_{t}^{2}\right), \\
& \beta^{u} \approx 0.076 b+2.32 \operatorname{cov}\left(b_{t}, g_{t}\right)+493.83 \operatorname{cov}\left(b_{t}, s_{t}^{2}\right) .
\end{aligned}
$$

The remaining quantities in these equations are unobservable. Therefore, any unconditional proxy alphas and betas are consistent with the conditional CAPM in principle. This is enough to contradict the conclusions in Lewellen and Nagel (2006). However, the result is also undesirable from the perspective of retaining the CAPM as a scientific model, as discussed in the introduction: It is unclear if a formal hypothesis test based on these equations can be created. Yet, it is possible to discuss the plausibility of these unobserved parameters, implied by values that are observable. This replicates the analysis that Lewellen and Nagel 
(2006) use to reject the theory in the conditional CAPM. But first, I explain that the other test proposed by Lewellen and Nagel (2006), based on short-window estimations, cannot be used to test the conditional CAPM, either.

\subsection{Short-window betas are also biased}

One necessary step to evaluate the covariances in Eq. (13) and Eq. (14) is to obtain conditional betas on the true market portfolio. This is sometimes done based on daily data, for example, and under the assumption that short-window regressions produce unbiased estimates of the true conditional alphas and betas. This is the second approach employed by Lewellen and Nagel (2006) to reject the theory in the conditional CAPM.

Apart from econometric difficulties in these estimations, a high frequency proxy beta is just an unconditional proxy beta obtained from a short sample. Hence, we can analyze it based on Eq. (8). Between 1 January 1964 and 1 January 2001, the average daily excess return on the CRSP portfolio is $g_{d}=\gamma_{d}=0.024 \%$ and its standard deviation is $\sigma_{d, P}=0.87 \%{ }^{6}$ This implies that $\frac{\gamma_{d}}{\sigma_{d, P}^{2}} \approx 3.14$ and $\frac{1}{\sigma_{d, P}^{2}} \approx 13337$. If we continue to assume $\frac{\sigma_{M}^{2}}{\sigma_{P}^{2}}=0.076$, the daily unconditional proxy beta is approximated by

$$
\beta_{d}^{u} \approx 0.076 b+3.14 \operatorname{cov}\left(b_{t}, g_{t}\right)+13337 \operatorname{cov}\left(b_{t}, s_{t}^{2}\right),
$$

which shows that the procedure does not produce an unbiased estimate of the conditional beta (nor the expected conditional beta, $b$ ). Indeed, the sources of bias are the same that affect proxy betas estimated in long samples in Eq. (14). However, there are possible differences: First, if the last covariance term, $\operatorname{cov}\left(b_{t}, s_{t}^{2}\right)$, is not at least 27 times smaller in daily frequency than it is in monthly frequency, the bias associated with this term is relatively larger in short-window proxy betas. In addition, if the idiosyncratic volatility portion of the market proxy volatility is larger in higher frequencies, lowering $\frac{\sigma_{M}^{2}}{\sigma_{P}^{2}}$, the attenuation bias is also more severe for short-window proxy betas. On the other hand, the bias from

\footnotetext{
${ }^{6}$ I consider the period between 1964 and 2001 because this is the period used in Lewellen and Nagel (2006).
} 
the first covariance term, $\operatorname{cov}\left(b_{t}, g_{t}\right)$, could be less severe for short-window proxy betas, given that this covariance could be substantially smaller in daily frequency than in monthly frequency. In summary, unconditional market proxy betas provide biased estimates of true betas regardless of the frequency of the data used in the estimation. Short-window betas (Lewellen and Nagel, 2006) cannot provide estimates of true conditional betas. Indeed, they are not even guaranteed to provide better estimates than betas estimated in longer samples.

\subsection{A first order magnitude adjustment}

As explained above, one of several issues with market proxy betas in any frequency is that they are heavily attenuated. According to Eq. (14), the magnitude of the instantaneous market proxy beta can be related to the true market beta (without the covariance terms) by

$$
b_{t} \sim \frac{1}{0.076} \beta_{t}
$$

where $~$ means "same order of magnitude". The equation suggests that estimates based on market betas must, at the very minimum, be adjusted for this difference in magnitude. In particular, the first order adjustment in Eq. (16) yields

$$
\begin{aligned}
& \operatorname{cov}\left(b_{t}, g_{t}\right) \sim \frac{\operatorname{cov}\left(\beta_{t}, \gamma_{t}\right)}{0.076}, \\
& \operatorname{cov}\left(b_{t}, s_{t}^{2}\right) \sim \frac{\operatorname{cov}\left(\beta_{t}, \sigma_{t}^{2}\right)}{0.076} .
\end{aligned}
$$

Lewellen and Nagel (2006) (Table 6, Panel B) provide details of possible values for $\operatorname{cov}\left(\beta_{t}, \gamma_{t}\right)$ involving value, size, and momentum portfolios: Their intervals range from $-0.12 \%$ to $-0.08 \%$ for momentum, $0.00 \%$ to $0.03 \%$ for value, and $-0.10 \%$ to $0.02 \%$ for 
size portfolios. Based on Eq. (17), the adjusted plausible intervals for momentum, value, and size portfolios, respectively, become

$$
\begin{aligned}
& \operatorname{cov}\left(b_{m o m, t}, g_{t}\right) \in[-1.59 \%,-1.06 \%] \\
& \operatorname{cov}\left(b_{h m l, t}, g_{t}\right) \in[0.00 \%, 0.40 \%] \\
& \operatorname{cov}\left(b_{s m b, t}, g_{t}\right) \in[-1.32 \%, 0.26 \%]
\end{aligned}
$$

Lewellen and Nagel (2006) also report that the covariances between conditional proxy betas and volatility, $\operatorname{cov}\left(\beta_{t}, \sigma_{t}^{2}\right)$, are between $-0.02 \%$ and $0.02 \%$ for every portfolio. Based on Eq. (18), this corresponds to

$$
\operatorname{cov}\left(b_{t}, s_{t}^{2}\right) \in[-0.26 \%, 0.26 \%]
$$

In addition, Fama and French (1992) (Table 2) estimate that the unconditional proxy betas for beta-sorted decile portfolios are between 0.79 and 1.73. Regardless of the issues with these unconditional betas, these values suggest approximate limits for the market proxy betas of long-short portfolios in general, such as size, value, and momentum portfolios:

$$
\left\|\beta_{t}\right\| \leq 0.94=1.73-0.79
$$

where $\|$.$\| denotes absolute value.$

These restrictions on the parameters of Eq. (13) and Eq. (14) finally mean that the equations produce (at least qualitatively) rejectable implications of the conditional CAPM theory that are internally consistent. Next, I show that these restrictions are consistent with the empirical evidence.

\subsection{The conditional CAPM explains asset pricing anomalies}

Fig. 1 shows combinations of covariances in Eq. (8) and Eq. (12) (in percentage terms) that generate monthly unconditional proxy alphas of $1.01 \%$ and $0.59 \%$ for momentum 
and value, respectively, (Lewellen and Nagel, 2006) and 0.07\% for size. In addition, they jointly generate unconditional proxy betas of $-0.5,-0.25$, and 0.21 , respectively, satisfying the parameter restrictions in the previous section. ${ }^{7}$ This contradicts the claim that the conditional CAPM is unable to explain these anomalies based on plausible assumptions and that the theory should be rejected (Lewellen and Nagel, 2006).
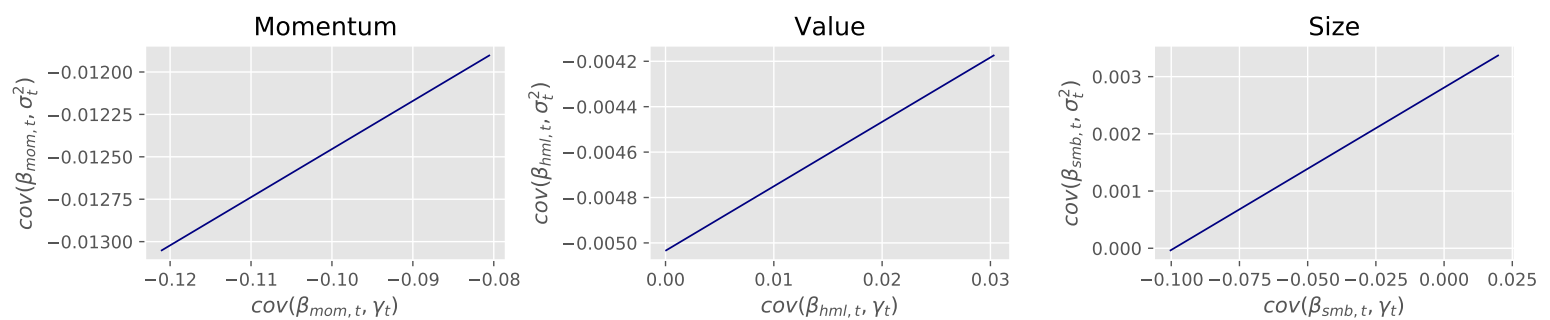

Figure 1: Combinations of covariances that generate the unconditional proxy alpha and beta of each portfolio.

Covariances between the conditional market proxy betas and the market proxy returns are on the horizontal axis, $\operatorname{cov}\left(\beta_{t}, \gamma_{t}\right)$. Covariances between the conditional market proxy betas and the variance of returns on the market proxy are on the vertical axis, $\operatorname{cov}\left(\beta_{t}, \sigma_{t}^{2}\right)$. The line in each graph shows the combinations of covariances that are consistent with the unconditional proxy betas and alphas of each respective portfolio: -0.5 and $1.01 \%$ for momentum, -0.25 and $0.59 \%$ for value, and 0.21 and $0.07 \%$ for size. All values are in percentage terms.

Summary: The figure shows combinations of plausible parameters that are consistent with each of the three premiums. Hence, it is impossible to reject the hypothesis that the conditional CAPM explains all of them.

The first two columns in Table 1 display the lower and upper bounds for the expected beta of each portfolio. To ease interpretation, I report the market proxy beta counterparts that correspond to the true betas (as if the betas were constant). This table complements Fig. 1. The values in this interval are consistent with the joint solution of Eq. (13) and Eq. (14), given the respective unconditional alpha and beta of the portfolio in the two right-most columns, and also the magnitude restrictions on $\operatorname{cov}\left(b_{t}, g_{t}\right)$ and $\operatorname{cov}\left(b_{t}, s_{t}^{2}\right)$ in Eq. (19) to Eq. (22). The expected beta interval is also consistent with the magnitude restriction on the betas in Eq. (23). Hence, the table shows that every unconditional beta

\footnotetext{
${ }^{7}$ I estimate all betas and the alpha for the size portfolio with their respective regressions on the market premium between 1964 and 2001 to match the period in Lewellen and Nagel (2006) with data from Kenneth French's data library. Momentum is the difference between the returns on winner and loser deciles, while size and value are the returns on the SMB and HML portfolios of Fama and French (1996).
} 
and alpha is consistent with the conditional CAPM. The remaining columns in Table 1 provide supplementary information: The two columns under $\operatorname{cov}\left(b_{t}, g_{t}\right)$ show bounds for the expected (proxy) beta such that the restrictions in Eq. (19) to Eq. (21) hold for each respective portfolio. The columns under $\operatorname{cov}\left(b_{t}, s_{t}^{2}\right)$ show similar bounds for Eq. (22).

Table 1: Bounds on the expected conditional (market proxy) betas for the value, size, and momentum portfolios.

\begin{tabular}{|c|c|c|c|c|c|c|c|c|}
\hline & \multirow[b]{2}{*}{$\beta \geq$} & \multirow[b]{2}{*}{$\beta \leq$} & \multicolumn{4}{|c|}{ Beta bounds implied by: } & \multicolumn{2}{|c|}{ Restrictions } \\
\hline & & & \multicolumn{2}{|c|}{$\operatorname{cov}\left(b_{t}, g_{t}\right)$} & \multicolumn{2}{|c|}{$\operatorname{cov}\left(b_{t}, s_{t}^{2}\right)$} & $\alpha^{u}$ & $\beta^{u}$ \\
\hline & 0.298 & 0.385 & 0.298 & 0.385 & -2.099 & 0.893 & $1.01 \%$ & -0.50 \\
\hline$\beta_{h n}$ & 0.012 & 0.077 & 0.012 & 0.077 & -1.801 & 1.192 & $0.59 \%$ & -0.25 \\
\hline$\beta_{\text {sm }}$ & -0.015 & 0.243 & -0.015 & 0.243 & -1.256 & 1.737 & $0.07 \%$ & 0.21 \\
\hline
\end{tabular}

The first two columns display the bounds for the expected conditional betas of the respective portfolios, the next (four) columns show the bounds implied individually by the magnitudes of the two covariances in Eq. (19) to Eq. (21) or Eq. (22), respectively, and the last columns contain the unconditional alphas and betas used in the calculations.

Summary: For any average conditional betas in the interval, Eq. (13) and Eq. (14) can be solved jointly and fulfill all restrictions for each premium. Hence, this table further confirms that it is impossible to reject the hypothesis that the conditional CAPM explains all premiums.

\subsection{Discussion}

The present paper contains a qualitative evaluation of the conditional CAPM, based on reasonable quantitative assumptions. This is equivalent to the main analysis in Lewellen and Nagel (2006). Yet, the conclusion is the opposite: The conditional CAPM explains asset pricing "anomalies" under plausible assumptions.

However, the paper does not contain a formal test of the conditional CAPM based on all the parameters in the model, such as expected conditional betas and the relevant covariance terms. Indeed, one of the main conclusions in the paper is that these parameters cannot be estimated, especially because short-window betas are also biased. In this sense, the paper 
confirms that conditional factor models are essentially untestable (Cochrane, 2005), except analytically, for example. ${ }^{8}$

In addition, the conclusion that the conditional CAPM explains the value, momentum, and possibly other anomalies does not include economic explanations for why value, momentum, and other portfolios have positive expected betas in the first place, and why their conditional betas change over time as they do. Although outside the scope of the present paper, this highlights the importance of this type of economic theory, similar to the conclusion in Ferson and Harvey (1991), for example. ${ }^{9}$ More broadly, the present paper directly contradicts the conclusions of the joint hypothesis tests of Lewellen and Nagel (2006), according to which "ignoring all variation in beta has little impact on asset-pricing tests", which would make economic explanations of this variation irrelevant.

\subsubsection{Not the Roll critique}

Roll (1977) notices that the market portfolio is not observable and that a rejection of the CAPM is simply a rejection of the proxy used for the market returns. As Campbell (2017) explains, the Roll critique in its complete form is a strong defense of the theory in the CAPM, but it also implies that the CAPM is not a scientific model since the theory is untestable: It implies that neither the conditional nor the unconditional CAPM can be rejected.

Clearly, the present paper is not a restatement of the Roll critique, as given by the restrictions used to test the conditional CAPM in Section 2.4. In addition, unlike the

\footnotetext{
${ }^{8}$ Another alternative in this case is to rely on (strong) assumptions about all state variables in the information sets of the individuals and to model conditional betas (Lettau and Ludvigson, 2001, for example).

${ }^{9}$ De Oliveira Souza (2020) offers this type of explanation for momentum, for example: He shows that intra-month momentum portfolios have positive (not negative) betas on average and theoretically explains why the series created by chaining returns on all these different portfolios together has a negative beta instead.
} 
conditional CAPM, the unconditional CAPM theory is rejected by the empirical evidence. Without variation in conditional betas, Eq. (13) and Eq. (14) become

$$
\begin{aligned}
& \alpha^{u \prime} \approx 0.43 \% b \\
& \beta^{u \prime} \approx 0.076 b .
\end{aligned}
$$

Based on these equations, it is impossible to match any of the pairs of unconditional proxy alphas and betas displayed in Table 1. For size, it can be argued that the estimates are noisy and the positive signs of both estimates in Eq. (24) and Eq. (25) are consistent with each other. However, value portfolios (after 1960) and especially momentum and the other 10 portfolios in Stambaugh et al. (2012) are inconsistent with this version of the model. All of them have negative unconditional proxy betas and positive proxy alphas that are impossible to accommodate simultaneously by Eq. (24) and Eq. (25). In summary, simply relaxing the empirical assumption that the CRSP index is the true market portfolio does not render the CAPM untestable.

Indeed, it is possible to use managed portfolios that take less risk when volatility is high (Moreira and Muir, 2017) to provide a concrete example of the issues that arise in unconditional CAPM tests: Apart from the attenuation bias, the negative covariance between risk and volatility, $\operatorname{cov}\left(b_{t}, s_{t}^{2}\right)<0$, implies that the unconditional proxy alphas of these managed portfolios are (even more) overestimated in Eq. (12), while their unconditional proxy betas are (even more) underestimated in Eq. (8). Inference based on unconditional proxy alphas alone, as in Moreira and Muir (2017), becomes even less informative to reject a conditional model in this case.

\section{Conclusion}

In the present paper we learn that the conditional CAPM tests proposed by Lewellen and Nagel (2006) are based on the joint (and internally inconsistent) hypothesis that the CAPM theory holds and that the CRSP index is the market portfolio on the MVF. Given that the 
CRSP portfolio is not on the MVF, this joint hypothesis is false even if the theory in the CAPM holds. In order to circumvent this problem, we learn how to create a test that is internally consistent with the hypothesis that the CAPM theory holds.

Based on this test, we learn that the conditional CAPM can explain the unconditional alphas and betas of value, momentum, and size portfolios. More generally, the paper argues that other anomalies might be explained in a similar way. Candidates include the returns on volatility managed portfolios, such as the ones in Moreira and Muir (2017), and portfolios typically believed to be associated with mispricing, such as the ones in Stambaugh et al. (2012). Most importantly - in sharp contrast with the previous consensus (Lewellen and Nagel, 2006) - we learn that theories that explain the evolution of conditional betas economically, and their relation with conditional market returns and volatility, have a good chance at explaining these "anomalies".

Finally, the paper is not a restatement of the Roll critique. For example, the unconditional version of the CAPM is empirically rejected based on the existence of portfolios with negative unconditional betas and positive alphas.

\section{A Appendix}

This is the derivation of a stock's unconditional covariance with the market premium, exactly as in Lewellen and Nagel (2006). Let $b_{t}=b+\eta_{t}$, where $\eta_{t}$ is the zero-mean, time-varying component of the conditional beta. According to the conditional CAPM, $R_{i, t}=b_{t} R_{M t}+e_{t}$, so the unconditional covariance between $R_{i, t}$ and $R_{M, t}$ is

$$
\begin{aligned}
\operatorname{cov}\left(R_{i, t}, R_{M, t}\right) & =\operatorname{cov}\left[\left(b+\eta_{t}\right) R_{M, t}, R_{M, t}\right] \\
& =b s_{M}^{2}+\mathrm{E}\left[\eta_{t} R_{M, t}^{2}\right]-\mathrm{E}\left[\eta_{t} R_{M, t}\right] \mathrm{E}\left[R_{M, t}\right]
\end{aligned}
$$


Given that $\mathrm{E}\left[\eta_{t}\right]=0, \mathrm{E}_{t-1}\left[R_{M, t}\right]=g_{t}, \mathrm{E}_{t-1}\left[R_{M t}^{2}\right]=g_{t}^{2}+s_{t}^{2}$, and $\mathrm{E}\left[R_{M, t}\right]=g$, the second term equals $\operatorname{cov}\left(\eta_{t}, g_{t}^{2}+s_{t}^{2}\right)$ and the last term equals $g \operatorname{cov}\left(\eta_{t}, g_{t}\right)$. Therefore, Eq. (A.2) becomes

$$
\operatorname{cov}\left(R_{i, t}, R_{M, t}\right)=b s_{M}^{2}+\operatorname{cov}\left(\eta_{t}, s_{t}^{2}\right)+\operatorname{cov}\left(\eta_{t}, g_{t}^{2}\right)-\gamma \operatorname{cov}\left(\eta_{t}, g_{t}\right)
$$

Next, substitute $g_{t}=g+\left(g_{t}-g\right)$ into the second-to-last term of Eq. (A.3) and simplify,

$$
\operatorname{cov}\left(R_{i, t}, R_{M, t}\right)=b s_{M}^{2}+\operatorname{cov}\left(\eta_{t}, s_{t}^{2}\right)+\gamma \operatorname{cov}\left(\eta_{t}, g_{t}\right)+\operatorname{cov}\left[\eta_{t},\left(g_{t}-g\right)^{2}\right]
$$

Finally, given that $b_{t}=b+\eta_{t}$, replace $\eta_{t}$ with $b_{t}$ to obtain the unconditional covariance between $R_{i, t}$ and $R_{M, t}$ in terms of $b_{t}$.

\section{References}

Acharya, Viral V., and Lasse Heje Pedersen, 2005, Asset pricing with liquidity risk, Journal of Financial Economics 77, 375-410.

Andrei, Daniel, Julien Cujean, and Mungo Wilson, 2018, The lost capital asset pricing model, CEPR Discussion Papers 12607.

Ang, Andrew, Joseph Chen, and Yuhang Xing, 2006, Downside risk, Review of Financial Studies 19, 1191-1239.

Atanasov, Victoria, Stig V. Moller, and Richard Priestley, 2020, Consumption fluctuations and expected returns, Journal of Finance forthcoming.

Bali, Turan G., 2008, The intertemporal relation between expected returns and risk, Journal of Financial Economics 87, 101-131.

Bali, Turan G., Robert F. Engle, and Yi Tang, 2017, Dynamic conditional beta is alive and well in the cross section of daily stock returns, Management Science 63, 3760-3779. 
Boguth, Oliver, Murray Carlson, Adlai Fisher, and Mikhail Simutin, 2011, Conditional risk and performance evaluation: Volatility timing, overconditioning, and new estimates of momentum alphas, Journal of Financial Economics 102, 363 - 389.

Campbell, John Y., 2017, Financial decisions and markets: A course in asset pricing (Princeton University Press).

Campbell, John Y., Jens Hilscher, and Jan Szilagyi, 2008, In search of distress risk, Journal of Finance 63, 2899-2939.

Choi, Jaewon, 2013, What drives the value premium?: The role of asset risk and leverage, Review of Financial Studies 26, 2845-2875.

Cochrane, John H., 2005, Asset Pricing, revised edition (Princeton University Press, Princeton).

Cooper, Michael J., Huseyin Gulen, and Michael J. Schill, 2008, Asset growth and the cross-section of stock returns, Journal of Finance 63, 1609-1651.

Daniel, Kent, and Sheridan Titman, 2006, Market reactions to tangible and intangible information, Journal of Finance 61, 1605-1643.

de Oliveira Souza, Thiago, 2020, A critique of momentum anomalies, Unpublished working paper. University of Southern Denmark.

Fama, Eugene F, and Kenneth French, 2006, Profitability, investment and average returns, Journal of Financial Economics 82, 491-518.

Fama, Eugene F, and Kenneth R. French, 1992, The cross-section of expected stock returns, Journal of Finance 47, 427-465.

Fama, Eugene F, and Kenneth R. French, 1996, Multifactor explanations of asset pricing anomalies, Journal of Finance 51, 55-84. 
Farago, Adam, and Roméo Tédongap, 2018, Downside risks and the cross-section of asset returns, Journal of Financial Economics 129, 69-86.

Ferson, Wayne E., and Campbell R. Harvey, 1991, The variation of economic risk premiums, Journal of Political Economy 99, 385-415.

Ferson, Wayne E., and Campbell R. Harvey, 1999, Conditioning variables and the cross section of stock returns, Journal of Finance 54, 1325-1360.

Ghosh, Anisha, Christian Julliard, and Alex P. Taylor, 2016, An information-theoretic asset pricing model, Working paper, London School of Economics.

Harvey, Campbell R., 1989, Time-varying conditional covariances in tests of asset pricing models, Journal of Financial Economics 24, 289 - 317.

Hasler, Michael, and Charles Martineau, 2019, Does the CAPM hold? A time-series perspective, Unpublished working paper. available at ssrn 3353903.

Hirshleifer, David, Kewei Hou, Siew Hong Teoh, and Yinglei Zhang, 2004, Do investors overvalue firms with bloated balance sheets?, Journal of Accounting and Economics 38, 297-331.

Jagannathan, Ravi, and Zhenyu Wang, 1996, The conditional CAPM and the cross-section of expected returns, Journal of Finance 51, 3-53.

Jegadeesh, Narasimhan, and Sheridan Titman, 1993, Returns to buying winners and selling losers: Implications for stock market efficiency, Journal of Finance 48, 65-91.

Kandel, Shmuel, and Robert F. Stambaugh, 1995, Portfolio inefficiency and the cross-section of expected returns, Journal of Finance 50, 157-184.

Lettau, Martin, and Sydney Ludvigson, 2001, Resurrecting the (C)CAPM: A cross-sectional test when risk premia are time-varying, Journal of Political Economy 109, 1238-1287. 
Lettau, Martin, Matteo Maggiori, and Michael Weber, 2014, Conditional risk premia in currency markets and other asset classes, Journal of Financial Economics 114, 197-225.

Lewellen, Jonathan, and Stefan Nagel, 2006, The conditional CAPM does not explain asset-pricing anomalies, Journal of Financial Economics 82, 289-314.

Loughran, Tim, and Jay R. Ritter, 1995, The new issues puzzle, Journal of Finance 50, $23-51$.

Lustig, Hanno N., and Stijn G. Van Nieuwerburgh, 2005, Housing collateral, consumption insurance, and risk premia: An empirical perspective, Journal of Finance 60, 1167-1219.

Moreira, Alan, and Tyler Muir, 2017, Volatility-managed portfolios, Journal of Finance 72, $1611-1644$.

Novy-Marx, Robert, 2013, The other side of value: The gross profitability premium, Journal of Financial Economics 108, 1-28.

Ohlson, James A., 1980, Financial ratios and the probabilistic prediction of bankruptcy, Journal of Accounting Research 18, 109-131.

Petkova, Ralitsa, and Lu Zhang, 2005, Is value riskier than growth?, Journal of Financial Economics 78, 187-202.

Piazzesi, Monika, Martin Schneider, and Selale Tuzel, 2007, Housing, consumption and asset pricing, Journal of Financial Economics 83, 531-569.

Roll, Richard, 1977, A critique of the asset pricing theory's tests Part I: On past and potential testability of the theory, Journal of Financial Economics 4, 129-176.

Roll, Richard, and Stephen Ross, 1994, On the cross-sectional relation between expected returns and betas, Journal of Finance 49, 101-21. 
Santos, Tano, and Pietro Veronesi, 2006, Labor income and predictable stock returns, Review of Financial Studies 19, 1-44.

Schweder, Tore, 2001, Statistical methods, history of: Post-1900, in Neil J. Smelser, and Paul B. Baltes, eds., International Encyclopedia of the Social \& Behavioral Sciences, 1503115037 (Pergamon, Oxford).

Sloan, Richard G., 1996, Do stock prices fully reflect information in accruals and cash flows about future earnings?, Accounting Review 71, 289-315.

Stambaugh, Robert, Jianfeng Yu, and Yu Yuan, 2012, The short of it: Investor sentiment and anomalies, Journal of Financial Economics 104, 288-302.

Stambaugh, Robert F, 1982, On the exclusion of assets from tests of the two-parameter model, Journal of Financial Economics 10, 237-268.

Titman, Sheridan, K. C. John Wei, and Feixue Xie, 2004, Capital investments and stock returns, Journal of Financial and Quantitative Analysis 39, 677-700.

Wang, Kevin Q., 2003, Asset pricing with conditioning information: A new test, Journal of Finance 58, 161-196. 